\title{
Zum Verhältnis von Sätzen und Textsorten - Eine korpusbasierte Analyse
}

\author{
Asmaa Mongi Saleh Bayoumi \\ Übersetzerin, Minia Universität \\ mongyasmaa@yahoo.com
}

Received: August 12, 2020 Accepted: October 11, 2020 Published: October 26, 2020

\begin{abstract}
Die vorligende Arbeit hat das Verhältnis von Sätzen und Textsorten durch die Analyse einiger Beispiele von Textsorten wie Wetterbericht, Kochrezept und Arztformular zum Untersuchungsstand. Es gibt verschiedene Textsorten wie Brief, Bericht, Rezept, Formular, Vertrag, Tagesbuch usw. Jeder Text hat seine eigene Besonderheiten, durch sie er sich von anderen Textsorten unterscheidet. Es gibt also vielfältige Satzarten wie Aussagesatz, Fragesatz, Aufforderungssatz, Ausrufesatz, Wunschsatz und unvollständiger Satz wie Ellipse. Jeder Satz hat auch seine eigene semantische und syntaktische Eigenschaften und verscheidene Funktionen. Deshalb wird das Verhältnis von Sätzen und Textsorten untersucht und behandelt. Gibt es eine Beziehung zwischen den Textsorten und den Sätzen, in denen sie verwendete werden? Daher befindet sich die Bedeutung der Forschung. Um die Ergebnise dieser Beziehung kennenzulernen, werden drei verschiedene Textsoren wie Wetterbericht, Kochrezept und Arztformular als ein Korpus dieser Arbeit analysiert. Wetterberichte gibt uns Informationen über das Wetter an, bei Kochrezept gibt es eine Erklärung wie man ein Gericht vorbereiten kann, dadurch gibt es eine Beratungen und Empfehlungen und das Arztformular enthält verschiedene Teile, die sich von den Patienten und ihren Krankheiten abängen.
\end{abstract}

Keywords: Begriffsatz, Sprechhandlung, Satzarten, Satzbau, Ellipse, Textsorten 


\section{Einleitung}

Der vorliegende Beitrag hat das Verhältnis von Sätzen und Textsorten zum Untersuchungsgegenstand, d. h. es wird untersucht, ob es eine Beziehung zwischen der Natur der Texte und den verwendeten Sätzen gibt oder nicht. Dabei werden der Begriff „Satz“ sowie die verschiedenen Satzarten (Aussagesatz, Fragesatz, Ausrufesatz, Aufforderungssatz, Wunschsatz), Satzbau (einfach oder komplex), Ellipse usw. erläutert. Dann kommt die Analyse einiger Textsorten wie Kochrezept, Formulare (Arztformulare) und Wetterbericht.

Die Arbeit versucht folgende Fragen zu beantworten:

- Gehören bestimmte Satzstrukturen zu bestimmten Textsorten? Zum Beispiel: Kochrezepte, Wetterberichte, Arztformulare.

- Welche Funktionen haben bestimmte Satzarten wie Aussagesätze, Fragesätze, Aufforderungssätze usw. in bestimmten Textsorten?

Die vorliegende Arbeit hat das Ziel, den Zusammenhang zwischen den Textsorten und den in diesen Texten verwendeten Sätzen zu zeigen, d. h., dass folgende Fragen beantwortet werden sollen, nämlich: Setzt die Textsorte eine bestimmte Art von Sätzen voraus? Oder kommen bestimmte Sätze in bestimmten Textsorten häufig vor?

Das Korpus der Arbeit besteht aus drei verschiedenen Textsorten. Diese umfassen fünf Wetterberichte, fünf Kochrezepte und fünf Arztformulare, die analysiert werden, indem auf die Satzarten und den Satzbau der Sätze genauer eingegangen wird.

\section{Forschungsstand}

Es gibt viele Beiträge, die sich mit dem Thema der komplexen Sätze, Ellipse, Satzarten, Frageformen usw. beschäftigen. Beispiele dafür sind die Werke von Christa Dürrscheid (2007) ,,Syntax. Grundlagen und Theorien“, Christa Dürrscheid (2006) „Einführung in die Schriftlinguistik", Karin Pittner und Judith Bermann (2007) „Deutsche Syntax. Ein Arbeitsbuch“, Andreas, Patrzek (2015) „Professionelle Fragetechnik für Führungskräfte, Berater und Coaches “, Uršula Krevs, Birk (2006) Argumentellipse: Aktantenweglassung in deutschen und slowenischen Reportagetexten.

Dürrscheid (2007) untersucht wichtige Begriffe wie Satz, Satzreihen und 
Satzgefüge. Sie erklärt, was man unter Matrixsatz (Hauptsatz) und Nebensatz versteht, was Parataxe und Hypotaxe sind, dann folgt eine Erklärung der semantischen und syntaktischen Subklassifikation von Nebensätzen. Nebensätze können verschiedene Bedeutungen haben wie Temporalsätze, Kausalsätze, Relativsätze, Finalsätze, Konditionalsätze oder Modalsätze usw. Danach findet man eine Angabe der Satzarten wie Aussagesatz, Fragesatz, Wunschsatz, Ausrufesatz und Aufforderungssatz.

Pittner \& Bermann (2007) behandeln viele Punkte, die sich auf den Satz beziehen. Das siebte Kapitel dieses Buchs trägt den Titel komplexe Sätze und bietet eine umfassende Erklärung des Aufbaus von komplexen Sätzen, und zwar Parataxe und Hypotaxe. Es werden also Formen der Nebensätze wie Relativsätze, eingebettete Interrogativsätze, Konjunktionalsätze und ihre syntaktischen Funktionen dargestellt. Man findet aber auch andere weitere Punkte wie Adverbialsatz, die Unterscheidung von Relativ-, Interrogativ- und Konjunktionalsätzen und infiniten Sätzen.

Uršula Krevs (2006) erklärt den Begriff Ellipse, dann gibt sie die verschiedenen Arten von Ellipsen wie Subjektellipse, Dialogellipse, Ellipse an, die für bestimmte Textsorten wie Kochrezept, Wetterbericht u.a. typisch sind.

Der vorliegende Beitrag versucht zu erklären, ob es eine Beziehung zwischen den Textsorten und den verwendeten Sätzen gibt, d. h. ob die Textsorte einen Einfluss auf die Art der verwendeten Sätze oder nicht hat.

\section{Theoretische Grundlagen}

\subsection{Der Begriff Satz}

Im Deutschen findet man verschiedene Definitionen vom Satz. Einige davon sind wie folgt:

„Im Allgemeinen aus mehreren Wörtern bestehende, in sich geschlossene, eine Aussage, Frage oder Aufforderung enthaltende sprachliche Einheit (...)“. (Duden, 2007, unter Satz)

Einen Satz kann man als eine sprachliche kommunikative unabhängige Einheit betrachten. Für eine kommunikative Abhängigkeit soll ein Satz eine vollkommene Sprechhandlung darstellen (vgl. Wachtel, 2005, 
S. 141). Das bedeutet, dass ein Satz nicht nur aus Wörtern besteht, die nacheinander vorkommen, ohne eine sinnvolle Bedeutung zu geben, sondern man soll davon etwas verstehen.

\section{Beispiele dafür:}

- Peter kann nicht mehr heute kommen, weil er viel zu tun hat.

- Der Schüler studiert sehr viel, damit er gute Noten bekommt.

In diesen Sätzen gibt es eine vollständige Sprechhandlung und eine sinnvolle Bedeutung.

Beispiele für Äußerungen, die nicht als Sätze betrachteł werden können, sind die folgenden:

- „Komme schon“.

- „Nein“.

- „Warum nicht“. (vgl. Wachtel, 2005, S. 141).

„Ein Satz besteht also aus einer Reihe von Wörtern, die eine Sprechhandlung ausdrücken“ (Mackowiak, 1999, 11). Das bedeutet, dass es innerhalb des Satzes eine Sprechhandlung gibt, wie Behauptungen, Warnung, Befehl, Wunsch, Aussage, Fragen, Aufforderungen, Empfehlung usw. (vgl. Mackowiak, 1999, S. 141).

Zusammenfassend kann man den Satz als eine sprachliche kommunikative Einheit definieren, die aus mehreren Wörtern besteht, die eine vollkommene Sprechhandlung wie Aussage, Frage, Aufforderung, Befehl, Drohung, Empfehlung usw. umfasst. Am Ende des Satzes gibt es gemäß seiner Art entweder ein Punkt, Fragezeichen oder Ausrufezeichen.

\subsubsection{Der Begriff Sprechhandlung}

Sprechakte oder Handlungsakte lassen sich in lokutionären Akt, illokutionären Akt und perlokutionären Akt zergliedern. Lokutionärer Akt wird auch Äußerungsakt genannt, in dem etwas geäußert wird (vgl. Franz, 2014, S. 28).

Der illokutionäre Akt gilt als der wichtigste Akt von Sprechakten; dadurch wird der lokutionäre Akt verstanden. Illokutionärer Akt bezeichnet die Inhaltsseite des lokutionären Aktes (Äußerungsakt), d. h. von dem Äußerungsakt kann man etwas verstehen, beispielsweise 
Drohung, Befehl, Ratschlag usw. (vgl. Hilbert, 2014, S. 7).

Hier wird gezeigt, dass der illokutionäre Akt sehr wichtig ist, weil davon man etwas verstehen kann. Illokutionäre Akt bezeichnet die Inhaltsseite des Satzes oder der Äußerung. Beispielsweise: Gib mir mein Buch oder wird ich dir nochmal nicht verleihen! Dieser Satz hat die Form vom Befehl, während der Satz eine Drohung bezeichnet. „Der perlokutionäre Teilakt bei Searle ist jene Handlung, mit der eine bestimmte Wirkung beim Adressaten erreicht wird, wie Überzeugung, Umstimmen, Einschüchtern, Beleidigen usw.“(Franz, 2014, S. 48).

- Waren Sie schon einmal in Paris? (Pelz, 2004, S. 243)

- Könntest du bitte deine Schuhe abputzen? (Pelz, 2004, S. 243)

- Dürfte ich Sie höflichst bitten, mir das Manuskript zurückzusenden? (Pelz, 2004, S. 243)

Diese drei Sätze haben die Form eines Fragesatzes, aber nur der erste Satz ist eine Frage, während der zweite Satz und der dritte Satz eine Aufforderung bezeichnen.

Im Folgenden werden verschiedene Satzarten erläutert.

\subsection{Satzarten}

Man unterscheidet zwischen verschiedenen Satzarten wie Aussagesatz, Fragesatz (Ergänzungsfragesatz - Entscheidungsfragesatz Alternativfragesatz), Ausrufesatz, Aufforderungssatz, Konditionalsatz und Wunschsatz.

Systematischer Zusammenhang von (durch bestimmte formale, grammatische Eigenschaften definierten) Satztypen zu spezifischen pragmatischen Funktionstypen. Als Satztypen gelten nach (traditioneller Grammatik) Aussagesatz, Frage-, Aufforderungs-,Wunsch- und Exklamativsatz. (Bussmann, 2002, unter Satzarten)

\subsubsection{Aussagesatz}

Aussagesatz bezeichnet einen von den Grundtypen von Satztypen. In diesen Sätzen befinden sich keine Fragewörter und das Verb kommt an der zweiten Stelle im Satz vor. Der Punkt ist der Satzendzeichen (vgl. Altmann, Hahnemann, 2007, S. 159).

Das Verb in Aussagesätzen kann nicht mehr im Imperativ vorkommen. Aussagesätze haben die Funktion von der Aussagen, Behauptungen, 
Vermutung oder Mitteilung (vgl. Mackowiak, 1999, S. 208).

\section{Beispiele:}

- Das Wetter ist sehr kalt.

- Monika kommt heute Abend.

\subsubsection{Fragesatz}

In diesem Satz fragt man nach etwas, worauf es eine Antwort gibt. Am Ende dieses Satzes steht ein Fragezeichen. Man unterscheidet zwischen zwei Typen von Fraagesätzen: Entscheidungsfragesatz und Ergänzungsfragesatz.

Beim Entscheidungsfragesatz steht das Verb an erster Stelle, das Verb kommt nicht im Imperativ vor. Als chrakteristische Funktion dieses Satzes, ist zu fragen, ob die Sachverhalte, die schon erwänt werden, zutreffen (vgl. Mackowiak, 1999, S. 209).

Beim Ergänzungsfragesatz steht das Verb im Gegensatz zum Entscheidungsfragesatz an der zweiten Stelle, denn an erster Stelle steht ein Fragewort wie (wo, wohin, warum, wann usw.).

Ergänzungsfragen haben die charakteristische kommunikative Funktion, danach zu fragen, ob ein Satzglied den durch die Frage wiedergegebenen Sachverhalt erfüllt oder ob ein Attribut eines Satzglieds diesen Sachverhalt erfüllt. (Mackowiak, 1999, S. 209)

A) Hat Fritz gestern ein Loch gegraben?“ (Ja / Nein)

(Entscheidungsinterrogativsatz) (Meibauer et al., 2013, 51)

B) Wann hat Fritz ein Loch gegraben? (Gestern)

(Ergänzungsinterrogativsatz) (Meibauer et al., 2013, 51).

\subsubsection{Aufforderungssatz}

Der Aufforderungssatz ist durch die Verbstellung und Imperativ als Verbmodus gekennzeichnet. Wichtig ist auch die Stellung des Verbs am Anfang des Satzes. Am Ende des Satzes findet man ein Ausrufezeichen (vgl. Dürrscheid, 2006, S. 154). Die typische und charakteristische Funktion dieses Satzes ist: ,jemanden zu etwas zu veranlassen.“ (Mackowiak, 1999, S. 209).

\section{$\underline{\text { Beispiele }}$}

- Bitte öffnen Sie die Tür! (Aufforderung) 
- Komm nicht zu spät! (Befehl) (Dürrscheid, 2006, S. 154).

- Komm mir nicht wieder mit demselben Märchen! (Mackowiak, 1999, S. 209).

\subsubsection{Wunschsatz}

Man unterscheidet zwischen realen und irrealen Wunschsätzen. In diesen Sätzen ist der Modus des Verbs Konjunktiv II. Beim irrealen Satz steht das Verb im Plusquamperfekt. In diesem Satz kann das Verb am Anfang oder am Ende des Satzes stehen. Als Satzzeichen kommt ein Punkt oder ein Ausrufezeichen (vgl. Dürrscheid, 2007, S. 66).

„Grammatisch begründeter Satztyp zum Ausdruck von Wünschen, der im Dt. durch Verb- Erststellung und Konjunktiv u.a. gekennzeichnet ist: Wäre diese Last doch endlich von mir genommen." (Bussmann, 2002, unter Wunschsatz)

\section{Beispiele:}

- Hätte ich viel Zeit!

- Käme er heute!

\subsubsection{Ausrufesatz}

Im Ausrufesatz (auch Exklamativsatz genannt) steht das Verb entweder an der ersten, zweiten Stelle oder am Ende des Satzes. Der Satzmodus dieses Satzes kann in der Form einer Aussage, Frage oder Aufforderung kommen. In diesem Satz wird die Emotion des Sprechers dargestellt. Die Intonation spielt eine Rolle bei dem Ausdruck der emotionalen Anteilnahme des Sprechers (vgl. Bussmann, 2002, unter Exklamativsatz).

z. B.: Das ist ja sehr schwer!

z.B.: Kommt sie heute nicht!

\subsection{Satzlänge}

Die Länge der Sätze kann durch die Zahl der Wörter gemessen wird. Das bedeutet, dass die Zahl der Wörter innerhalb eines Satzes die Länge des Satzes bestimmt (vgl. Köhler et al., 2005, S. 300).

Möslein (1974, S. 81) bestimmt die Satzlänge nach „,der Zahl der Wörter, die durchschnittlich pro Satz vorkommen (...)“ (Möslein, 1974, S. 81, z. nach Bär, 2007, S. 54).

\subsection{Satzbau}

Der Satz kann entweder Haupt- oder Nebensatz sein. Der Hauptsatz 
(auch Matrixsatz genannt) ist übergeordnet, denn er umfasst den

Nebensatz, d. h. der Nebensatz gilt als ein Satzglied in dem Hauptsatz, wie Klaus Welke erklärt. Deshalb ist der Nebensatz untergeordnet und eingebettet, denn er hat eine enge semantische Beziehung zum Hauptsatz. Ohne diese Beziehung zum Hauptsatz hat der Nebensatz keine sinnvolle Bedeutung. (vgl. Welke, 2007, S. 39).

Z. B.: Wenn ich Zeit habe, besuche ich meine Freundinnen. (Nebensatz + Hauptsatz)

Z.B.: Falls das Wetter schlecht ist, bleiben wir zu Hause. $\quad$ (Nebensatz + Hauptsatz)

Der Nebensatz kann temporal, lokal, kausal oder modal sein:

Tabelle 1: Nebensatztypen

\begin{tabular}{lll}
\hline \multicolumn{1}{c}{ Nebensatztyp } & \multicolumn{1}{c}{ Beispiele } & \multicolumn{1}{c}{ Quelle } \\
\hline Temporal & $\begin{array}{l}\text { Seitdem ich in Hamburg bin, habe } \\
\text { ich eine Erkältung. }\end{array}$ & $\begin{array}{l}\text { (Dreyer \& Schmitt, 2000, } \\
\text { S. 155) }\end{array}$ \\
\hline \multirow{2}{*}{ Lokal } & $\begin{array}{l}\text { Ich weiß nicht, wo meine Brille } \\
\text { ist? }\end{array}$ & $\begin{array}{l}\text { (Dreyer \& Schmitt, 2000, } \\
\text { S. 179) }\end{array}$ \\
\hline \multirow{2}{*}{ Kausal } & $\begin{array}{l}\text { Weil man starke Schneefälle } \\
\text { vorausgesetzt hatte, mussten wir } \\
\text { unsren Ausflug verschieben. }\end{array}$ & $\begin{array}{l}\text { (Dreyer \& Schmitt, 2000, } \\
\text { S. 158) }\end{array}$ \\
\hline Modal & $\begin{array}{l}\text { Er ist noch reicher, als ich } \\
\text { erwartet habe. }\end{array}$ & $\begin{array}{l}\text { (Dreyer \& Schmitt, 2000, } \\
\text { S. 169) }\end{array}$ \\
\hline
\end{tabular}

\subsubsection{Einfache und komplexe Sätze}

Die größte Einheit in der syntaktischen Beschreibung ist der Satz. Dabei kann es sich um einen einfachen, aber auch um einen komplexen Satz handeln. Ein komplexer Satz besteht aus mehreren Teilsätzen (...). (Pittner \& Berman, 2007, S. 96)

Die Einheit, die aus genau einem Verbalkomplex und den Komplementen des Verbs sowie gegebenfalls Supplementen besteht, die aber beide keine Satzstruktur enthalten, nennen wir „einfache Satzstruktur. (Pasch, Brauße, Breindl, Hermann Waßner, 2003, S. 84)

Verbalkomplex, Verbgruppe und Satzstruktur sind „Finite Ausdrücke“, die aus einem Subordinator und einem Verballetzsatz gebildet sind, und die traditionell als (Neben- oder Glied-) Sätze bezeichnet werden. (Pasch, Brauße, Breindl, Hermann Waßner., 2003, S. 48)

Im einfachen Satz befinden sich wenigstens ein Subjekt und ein Prädikat und es ist also erforderlich, dass ein finites Verb vorhanden ist. (vgl. 
Grein, 1998, S. 31).

Ein komplexer Satz besteht aus der Kombination mehrerer Sätze (sentences)oder eines Satzes mit mehreren Teilsätzen bzw. satzwertigen Konstruktionen (clauses). Die einzelnen Sätze und Teilsätze können sehr lose oder sehr eng miteinander verknüpft sein. (Grein, 1998, S. 32)

Ein komplexer Satz (auch zusammengesetzter Satz genannt) umfasst mehrere Sätze, die entweder miteinander verbunden oder lose sind. Beispielsweise: Peter kommt heute nicht, weil er sehr krank ist.(Verbindung durch die Subjunktion weil). Peter kommt heute nicht. Er hat viel zu tun. Die Sitzung kann deshalb heute nicht stattfinden. (Drei Sätze kommen ohne Verbindung nacheinander und sind von einander durch einen Punkt getrennt).

Sätze, die ihrerseits wieder aus Sätzen bestehen, nennt man zusammengesetzte Sätze, komplexe Sätze oder Perioden. Innerhalb einer Periode unterscheidet man zwischen Haupt- und Nebensätzen (Hentschel \& Weydt, 2013, S. 373).

\section{Beispiele für Hypotaxe und Parataxe:}

1. Franz kommt heute nicht, weil er viel zu tun hat. (Hypotaxe)

2. Sara studiert so viel, weil sie gute Noten bekommen will und damit sie später einen guten Beruf finden kann. (Hypotaxe)

3. Lisa liest einen Roman, spricht mit ihrer Schwester und isst ihr Mittagessen. (Parataxe)

4. Peter geht heute Abend ins Kino. Er will seine Freunde dort treffen. (Parataxe)

Ein komplexer Satz kann parataktisch oder hypotaktisch wie in den obengenannten Beispielen sein. Im ersten und zweiten Beispiel findet man einen Hauptsatz und einen Nebensatz, während im dritten und vierten Beispiel Hauptsätze miteinander durch die Konjunktion ,und““ und ein Komma verbunden sind.

\subsubsection{Die Parataxe}

Parataxe bezeichnet nach Duden im Gegensatz zur Hypotaxe die Nebenordnung von Sätzen oder Satzgliedern (vgl. Duden, 2007, unter Parataxe).

„Bei einer Parataxe (Satzreihung) werden strukturell gleichrangige Sätze verbunden. Dabei kann es sich um Hauptsätze handeln, aber auch um Nebensätze“ (Pittner \& Bermann, 2007, S. 96). 
Das bedeutet, dass wenn es auch Nebensätze gibt, die in einer Gleichrangigkeit sind, kann man sie auch als Parataxte bezeichnen.

Z. B. Sara studiert so viel, dass sie gute Noten bekommen kann und dass sie dann einen guten Beruf finden kann. (Zwei Nebensätze kommen in einer gleichrangigen Reihe)

\section{Beispiele für Parataxe:}

a) Eva weint und/ oder/ aber/ denn Frieda lacht. (syndetisch) (Pittner \& Bermann, 2007, S. 96)

b) Eva weint, trotzdem/ deshalb lacht Frieda. (syndetisch) (Pittner \& Bermann, 2007, S. 96)

c) Eva weint, Frieda lacht" (und Emil sagt gar nichts mehr). (asyndetisch) (Pittner \& Bermann, 2007, S. 96)

„Bei einer Parataxe (Satzreihung) werden strukturell gleichrangige Sätze verbunden. Dabei kann es sich um Hauptsätze handeln, aber auch um Nebensätze“ (Pittner \& Bermann, 2007, S. 96).

Das bedeutet, dass nicht nur Hauptsätze Parataxe aufweisen können, sondern auch Nebensätze, wenn sie gleichrangig sind.

\section{Beispiele dafür}

- Lisa studiert ihre Fächer und Lara spricht mit ihrer Mutter. (zwei Hauptsätze)

- Peter sagt, dass Lisa ihre Fächer studiert und dass Lara mit ihrer Mutter spricht. (Zwei Nebensätze kommen in einer gleichrangigen Reihe vor)

\subsection{Begriff Syndetisch}

„Syndetisch 〈Adj.〉 [griech.sýdetos= zusammengebunden] (Sprachw.): durch eine Konjunktion verbunden“ (Duden, 2007, unter syndetisch).

\section{Beispiele}

- Otto hat staubgesaugt und Anna (hat) aufgeräumt. (syndetisch, koordiniert) “ (Breindl, Volodina \& Hermann Waßner, 2014, S. 406)

- Otto hat staubgesaugt. Und Anna hat aufgeräumt. (syndetisch, parataktisch) “ (Breindl, Volodina \& Hermann Waßner, 2014, S. 406) Einfach kann man sagen, dass syndetisch oder Syndese Verbindung der Einheiten einer Satzreihe miteinander durch Konjunktion bedeutet (vgl. Adamzik, 2010, S. 197). 


\subsection{Begriff Asyndetisch}

Bei der Asyndese oder asyndetisch spricht man von der „Verbindung von sprachlichen Ausdrücken (Wörter, Syntagmen, Sätzen) ohne Konjunktion.“ (Bussmann, 2002, unter Asyndese).

\section{Beispiele}

- Ich kam, ich sah, ich siegte. (asyndetisch) (Adamzik, 2010, S. 197)

- Otto hat staubgesaugt. Anna hat aufgeräumt. (asyndetisch) (Breindl, Volodina \& Hermann Waßner, 2014, S. 406)

\subsubsection{Die Hypotaxe}

Unter Hypotaxe versteht man eine Unterordnung von Sätzen, d. h. ein Nebensatz ist einem Hauptsatz untergeordnet. Dadurch hat der Nebensatz allein keine selbständige sinnvolle Bedeutung, denn er ist sehr eng mit dem Hauptsatz verbunden. Der Hauptsatz ist übergeordnet, während der Nebensatz untergeordnet ist. Hier spricht man von subordinierenden Konjunktionen, wie weil, dass, ob, obwohl usw., die Haupt- und Nebensätze verbinden (vgl. Pittner \& Bermann, 2007, S. 96).

\section{Beispiele}

a) Monika hat uns mitgeteilt, dass sie heute nicht kommen kann. $(\mathrm{H}+\mathrm{N})$

b) Friedrich geht nach der Schule, ohne seine Tasche zu nehmen, weil er sie vergessen hat. $(\mathrm{H}+\mathrm{N}+\mathrm{N})$

Im Folgenden werden die Formen der komplexen Sätze und deren Struktur laut Pittner \& Bermann (2007) erläutert:

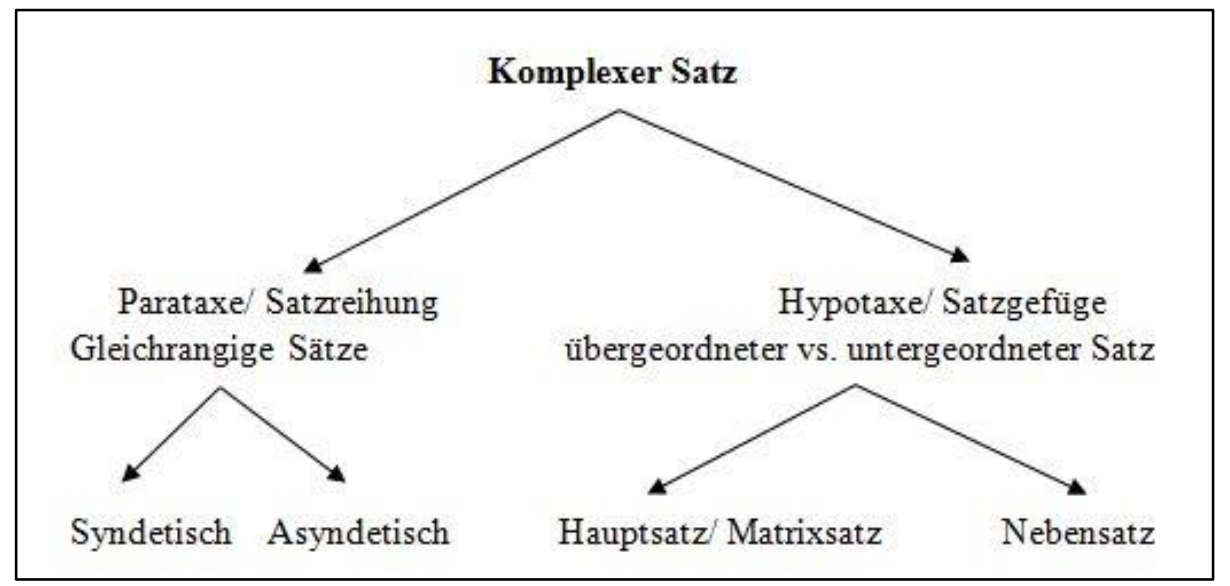

Abbildung 1: Aufbau komplexer Sätze (Pittner \& Bermann, 2007, S. 97) 


\subsection{Ellipse}

Die terminologische Bezeichnung „Ellipse“ stammt aus der Rhetorik und bedeutet im Griechischen (ellípsis) „Mangel“, im Lateinischen ist „ellipsis“ ein griechisches Lehnwort und bedeutet „Auslassung eines Wortes“ ( Birk, 2006, S. 23).

Es gibt verschiedene Formen von Ellipsen wie situative Ellipse wie Sie isst Banane und sie (isst) Apfel, Dialogellipse (Wann fährst du nach Kairo? Morgens), Textsortenellipse (Das bedeutet, dass Ellipse eine typische Eigenschaft für einige Textsorten ist. Diese Texte sind wie Telegramm, Kochrezept, Wetterbericht, Pressetext usw., Koordinationsellipse (Friedrich sieht fern und isst derweil), Vergleichsellipse (Lara studiert mehr als Sara), Subjektellipse (Fahre jetzt!) usw. (vgl. Birk, 2006, S. 25).

Es gibt also lexikalische Ellipsen, darunter befinden sich definite und indefinite Ellipsen. Bei den Imperativsätzen wird auch das Subjekt weggelassen wie (Bleibe zu Hause!). Lexikalische Ellipse ist wie „Die Hühner legen (Eier) “, definite Ellipse: „Er isst gerade“ Infinite Ellipse: „Jakob gestand endlich“ (Bussmann, 2002, unter Ellipse).

\subsection{Textsorten}

Als Textsorten gelten Klassen von Texten mit bestimmten strukturellen und funktionalen Merkmalen, die sich in konventionalisierten Mustern mit einer hohen Gebrauchshäufigkeit verfestigt haben. (Gläser, 1990, S. 28)

Textsorten spielen eine zentrale Rolle in der Textlinguistik. Sie tragen zur Erklärung der Beziehungen von kontextuellen, funktionalen und kommunikativen Handlungen und ihre Struktur bei (vgl. Gläser, 1990, S. 53).

Textsorten wie Wetterberichte, Rezepte, Kochrezepte, Vorträge, Briefe, Leserbriefe, Liebesbriefe, Gutachten, Tagebücher, Telegramme, Nachrichten, Erzählungen, Märchen, Romane, Kommentare usw. bezeichnen verschiedene Aspekte vom Leben, die entweder privat, offiziell oder öffentlich usw. sind (vgl. Gansel \& Jürgens, 2007, S. 54).

Textsorten sind konventionell geltende Muster für komplexe sprachliche Handlungen und lassen sich als jeweils typische Verbindungen von kontextuellen (situativen), kommunikativ- funktionalen und strukturellen (grammatischen und thematischen) Merkmalen beschreiben. (Brinker, 2010, S. 125)

Die Textsorten (...) helfen als konventionalisierte Rahmenvorgaben für die 
Kommunikation, das Mitteilungsanliegen in eine relativ vorgegebene Form zu fügen und andererseits das Mitgeteilte besser erwarten und schnell einordnen zu können. (Kalverkämper, 1996, S. 20)

Schließlich findet man, dass Textsorten eine Gruppe von Texten sind, die eine bestimmte Funktion und eine bestimmte Struktur haben. Textsorten bezeichnen auch eine Form der Zusammensetzung und Verbindung von kontextuellen, kommunikativen, strukturellen und funktionalen Merkmalen miteinander. Beispiele von Texten sind Kochrezept, Wetterbericht, Kommentar, Vortrag, Roman usw.

\section{Erstens: Wetterberichte}

Tabelle 2: Analyse von fünf Wetterberichten ${ }^{1}$

\begin{tabular}{|c|c|c|c|c|}
\hline \multirow[t]{2}{*}{ Der Texte } & \multirow{2}{*}{$\begin{array}{c}\text { Satzarten in } \\
\text { Wetterberichten }\end{array}$} & \multicolumn{3}{|c|}{ Satzstruktur } \\
\hline & & einfach & Hypotaxe & Parataxe \\
\hline Text 1 & \multirow{5}{*}{$\begin{array}{c}\text { Aussagesätze } \\
(100 \%)\end{array}$} & $(42,85 \%)$ & $(0 \%)$ & $(57,14 \%)$ \\
\hline Text 2 & & $(62,5 \%)$ & $(0 \%)$ & $(37,5 \%)$ \\
\hline Text 3 & & $(64,70 \%)$ & $(11,67 \%)$ & $(23,52 \%)$ \\
\hline Text 4 & & $(90 \%)$ & $(5 \%)$ & $(5 \%)$ \\
\hline Text 5 & & $(78,94 \%)$ & $(5,26 \%)$ & $(15,78 \%)$ \\
\hline \multicolumn{5}{|c|}{ Endstatistik der Textsorte (Wetterbericht) } \\
\hline Durchscnitt & $\begin{array}{c}\text { Aussagesätze } \\
(100 \%)\end{array}$ & $\begin{array}{c}\text { einfache } \\
\text { Sätze } \\
(67,79 \%)\end{array}$ & $\begin{array}{c}\text { Hypotaxe } \\
(4,40 \%)\end{array}$ & $\begin{array}{l}\text { Parataxe } \\
(27,78 \%)\end{array}$ \\
\hline
\end{tabular}

\section{Zweitens: Kochrezepte}

Die Textsorte „Kochrezepte“ sind kompliziert, da sie aus mehreren

Teilen bestehen. Diese Teile sind Zutaten, Wissenswert vom Rezept, Zeit vom Rezept, Zubereitung vom Rezept und Tipps zum Rezept.

Tabelle 3: Analyse von fünf Kochrezepten ${ }^{2}$

\begin{tabular}{clcccc}
\hline Texte & \multirow{2}{*}{$\begin{array}{c}\text { Satzarten in } \\
\text { Kochrezepten }\end{array}$} & \multicolumn{4}{c}{ Satzstruktur } \\
\cline { 3 - 5 } & & einfach & elliptisch & Hypotaxe & Parataxe \\
\hline Text 1 & $\begin{array}{l}\text { Aufforderungs- } \\
\text { sätze (60\%) }\end{array}$ & $(36 \%)$ & $(40 \%)$ & $4 \%$ & $20 \%$ \\
& $\begin{array}{l}\text { Aussagesätze } \\
(44,44 \%)\end{array}$ & & & & \\
\hline
\end{tabular}

${ }^{1}$ Diese Texte sind der Webseite „WetterOnline: Wetter aktuell, Wettervorhersage und Wetterbericht" entnommen.

${ }^{2}$ Diese Texte sind dem österreichischen Gourmet-Portal entnommen. 


\begin{tabular}{|c|c|c|c|c|c|}
\hline \multicolumn{2}{|c|}{ https://jltmin.journals.ekb.eg } & \multicolumn{3}{|c|}{ Journal of Languages and Translation (JLT) } & \multirow{2}{*}{$\frac{\text { Vol. 7, No. 1, 20 }}{(33,33 \%)}$} \\
\hline Text 2 & $\begin{array}{l}\text { Aufforderungs- } \\
\text { sätze }(55,55 \%) \\
\text { Aussagesätze } \\
(40 \%)\end{array}$ & $(22,22 \%)$ & $(44,44 \%)$ & $(0 \%)$ & \\
\hline Text 3 & $\begin{array}{l}\text { Aufforderungs- } \\
\text { sätze }(71,42 \%) \\
\text { Aussagesätze } \\
(25,57 \%)\end{array}$ & $(21,42 \%)$ & $(35,71 \%)$ & $(0 \%)$ & $(42,85 \%)$ \\
\hline Text 4 & $\begin{array}{l}\text { Aufforderungs- } \\
\text { sätze }(68,75 \%) \\
\text { Aussagesätze } \\
(31,25 \%)\end{array}$ & $(50 \%)$ & $(18,75 \%)$ & $(0 \%)$ & $(31,25 \%)$ \\
\hline Text 5 & $\begin{array}{l}\text { Aufforderungs- } \\
\text { sätze }(58,82 \%) \\
\text { Aussagesätze } \\
(31,25 \%)\end{array}$ & $(29,41 \%)$ & $(29,41 \%)$ & $(11,76 \%)$ & $(29,41 \%)$ \\
\hline \multicolumn{6}{|c|}{ Endstatistik der Textsorte (Kochrezept) } \\
\hline $\begin{array}{l}\text { Durch- } \\
\text { schnitt }\end{array}$ & $\begin{array}{l}\text { Aufforderungs- } \\
\text { sätze }(62,90 \%) \\
\text { Aussagesätze } \\
(37,8 \%)\end{array}$ & $\begin{array}{ll}\text { einfach } & \text { elliptisch } \\
(31,81 \%) & (33,60 \%)\end{array}$ & $\begin{array}{l}\text { Hypotaxe } \\
(3,15 \%)\end{array}$ & $\begin{array}{l}\text { Paratax } \\
(31,36 \%\end{array}$ & \\
\hline
\end{tabular}

\section{Drittens: Arztformulare}

Tabelle 4: Analyse von fünf Arztformularen ${ }^{3}$

\begin{tabular}{|c|c|c|c|c|c|}
\hline \multirow{2}{*}{ Texte } & \multirow{2}{*}{$\begin{array}{c}\text { Satzarten in } \\
\text { Arztformularen }\end{array}$} & \multicolumn{4}{|c|}{ Satzstruktur } \\
\hline & & einfach & elliptisch & Hypotaxe & Parataxe \\
\hline Text 1 & $\begin{array}{l}\text { Fragesätze } \\
\text { Entscheidungsfragen } \\
(71,42 \%) \\
\text { Ergänzungsfragen } \\
(28,57 \%)\end{array}$ & $(100 \%)$ & $(0 \%)$ & $(0 \%)$ & $(0 \%)$ \\
\hline Text 2 & $\begin{array}{l}\text { Fragesätze } \\
\text { Entscheidungsfragen } \\
(50 \%) \\
\text { Ergänzungsfragen } \\
(28,30 \%) \\
\text { Aufforderungssätze } \\
(7,69 \%)\end{array}$ & $(53,84 \%)$ & $(42,30 \%)$ & $(0 \%)$ & $(3,84 \%)$ \\
\hline
\end{tabular}

${ }^{3}$ Diese Texte sind der Webseiten der deutschen Rentenversicherung und desStadtspitals Waid und Triemli Zürich entnommen. 


\begin{tabular}{|c|c|c|c|c|c|}
\hline Text 3 & $\begin{array}{l}\text { Fragesätze } \\
\text { Entscheidungsfragen } \\
(0 \%) \\
\text { Ergänzungsfragen } \\
(85,71 \%) \\
\text { Aufforderungssätze } \\
(14,28 \%)\end{array}$ & $(14,28 \%)$ & $(85,71 \%)$ & $(0 \%)$ & $(0 \%)$ \\
\hline Text 4 & $\begin{array}{l}\text { Fragesätze } \\
\text { Entscheidungsfragen } \\
(0 \%) \\
\text { Ergänzungsfragen }(80 \%) \\
\text { Aufforderungssätze } \\
(20 \%)\end{array}$ & $(0 \%)$ & $(100 \%)$ & $(0 \%)$ & $(0 \%)$ \\
\hline Text 5 & $\begin{array}{l}\text { Fragesätze } \\
\text { Entscheidungsfragen } \\
(33,33 \%) \\
\text { Ergänzungsfragen } \\
(13,33 \%) \\
\text { Aussagesätze } \\
(40 \%) \\
\text { Aufforderungssätze } \\
(13,33 \%)\end{array}$ & $(53,33 \%)$ & $(40 \%)$ & $(6,66 \% \%)$ & $(0 \%)$ \\
\hline \multicolumn{6}{|c|}{ Endestatistik von Satzarten und Satzstruktur in der Textsorte (Arztformular) } \\
\hline $\begin{array}{l}\text { Durch- } \\
\text { schnitt }\end{array}$ & $\begin{array}{l}\text { Entscheidungsfragen } \\
(30,59 \%) \\
\text { Ergänzungsfragen } \\
(49,98 \%) \\
\text { Aufforderungssätze } \\
(11,06 \%) \\
\text { Aussagesätze } \\
(8 \%)\end{array}$ & $\begin{array}{c}\text { einfach } \\
(44,29 \%)\end{array}$ & $\begin{array}{l}\text { elliptisch } \\
(53,60 \%)\end{array}$ & $\begin{array}{c}\text { Hypotaxe } \\
(1,33 \%)\end{array}$ & $\begin{array}{c}\text { Parataxe } \\
(0,768 \%)\end{array}$ \\
\hline
\end{tabular}

\section{Zusammenfassung und Ergebnisse der Arbeit}

Die vorliegende Arbeit untersucht die Beziehung zwischen den Textsorten und den verwendeten Sätzen und die Funktionen dieser Satzarten in drei verschiedenen Textsorten, nämlich: Wetterbericht, Kochrezept und Arztformular. Um diese Beziehung herauszuarbeiten wurden einige Begriffe erläutert, dazu gehören Satz, die verschiedenen Satzarten (Aussagesatz, Fragesatz, Ausrufesatz, Aufforderungssatz, Wunschsatz), der Satzbau, Ellipse usw. Daraufhin wurde ein Korpus (drei verschiedene Textsorten) analysiert.

Die Ergebnisse zeigen, dass bestimmte Satzstrukturen zu bestimmten Textsorten gehören und auch dass bestimmte Satzarten in bestimmten Textsorten eigene Funktionen haben. Das wird durch die Analyse von 
den drei obengenannten Textsorten (Wetterbericht, Kochrezept und Arztformular) erklärt.

Erstens: Beim Wetterbericht sind die verwendeten Sätze Aussagesätze (100\%), weil dieser Texttyp uns Informationen gibt. Die Satzstruktur ist sowohl einfach $(67,79 \%)$ als auch komplex (Parataxe: 27,78\% und Hypotaxe: 4,40\%). Einfache Satzstruktur kommt aber häufiger als komplexe vor. Wetterbericht als eine der kommunikative Textsorte hat die Funktion etwas mitzuteilen oder auszusagen und das ist die Hauptfunktion von Aussagesätzen. Dadurch erklärt sich die Beziehung zwischen der Textsorte und der Funktion der verwendeten Sätzen, in dem vorhanden sind.

Zweitens: Beim Kochrezept gibt es Aufforderungssätze (62,90\%) und Aussagesätze (37,08\%), weil im Kochrezept Informationen mitgeteilt und Beratungen gegeben werden. Die Satzstruktur ist elliptisch $(33,60 \%)$, einfach $(31,81 \%)$ und komplex (Hypotaxe: $3,15 \%$ und Parataxe:

$31,36 \%)$. Kochrezept als eine Textsort ist am bischen schwer, denn diese Textsorte verschiedene Teile umfasst, wo man - wie in diesen drei Textsorten findet- Aufforderungssätze und Aussagesätze finden kann. Aufforderungssätze haben die Funktion die anderen zu etwas zu veranlassen, die anderen aufmerksam zu machen usw. Deshalb findet man, dass solche Sätze am häufigsten in den Texten vorkommen. Hier zeigt sich auch die Beziehung zwischen der Funktion von gebrauchten Sätzen und der Textsorte. Kochrezept kann also uns eine Information angeben, deshalb gibt es auch Aussagesätze neben den Aufforderungssätzen.

Drittens: Beim Arztformular gibt es Fragesätze (Ergänzungsfragesatz: 49,98\% - Entscheidungsfragesätze: 30,95\%), Aussagesätze (8\%) und Aufforderungssätze (11,06\%). Weil Arztformulare Informationen brauchen, sind die meisten verwendeten Sätze Fragesätze. Aussagesätze werden benutzt, um Informationen über das Formular anzugeben. Aufforderungssätze im Arztformular werden mit dem Ziel benutzt, Anweisungen beim Ausfüllen vom Formular zu beachten. Die Satzstruktur ist elliptisch (53,60\%), einfach (4,29\%) und komplex (Hypotaxe: 1,33\% und Parataxe: 0,768). Artztformulare sind auch am 
bischen schwer, denn sie aus vielen Teilen bestehen. Diese Teile

kommen in verschiedenen Satzarten vor, jede von denen bestimmte Funktion haben, wie Fragesätze (in erster Linie, ihre Funktion etwas zu wissen, eine Information zu bekommen), Aufforderungssätze (Beratungen angeben) und Aussagesätze (etwas mitteilen, Information angeben).

\section{Literaturverzeichnis}

Adamzik, K. (2004). Textlinguistik: Eine einführende Darstellung. Tübingen: Max Niemeyer Verlag.

Adamzik, K. (2010). Sprache: Wege zum Verstehen. Dritte, überarbeitete Auflage. Tübingen und Basel: Francke.

Bär, J. A., Roelcke, T., Steinhauer, A. (Hrsg.) (2007). Sprachliche Kürze: Konzeptuelle, strukturelle und pragmatische Aspekte. Berlin: Walter de Gruyter Verlag.

Birk, U. K. (2006). Argumentellipse: Aktantenweglassung in deutschen und slowenischen Reportagetexten. Tübingen: Narr.

Breindl, E., Volodina, A. \& Hermann Waßner, U. (2014). Handbuch der deutschen Konnektoren 2: Semantik der deutschen Satzverknüpfer. Berlin: de Gruyter.

Brinker, K. (2010). Linguistische Textanalyse: Eine Einführung in Grundbegriffe und Methoden. Berlin: Erich Schmidt Verlag.

Bussman, H. (2002). Lexikon der Sprachwissenschaft. 3., aktualisierte und erweiterte Auflage. Stuttgart: Alfred Kröner Verlag.

Dam, C. (2005). Die Sprechakttheorie nach Austin und Searle. München: Grin Verlag.

Duden (2007). Deutsches Universalwörterbuch. 6., überarbeitete Auflage. Mannheim: Duden Verlag.

Dürrscheid, C. (2006). Einführung in die Schriftlinguistik. 3., überarbeitete Auflage. Göttingen: Vandenhoeck und Ruprecht Verlag.

Dürrscheid, C. (2007). Syntax - Grundlagen und Theorien. 4., überarbeitete und ergänzte Auflage. Göttingen: Vadenhoeck und Ruprecht Verlag.

Franz, N. (2014). Die Sprechakttheorie nach Austin und Searle: Äußerungen als Handlung. Hamburg: Diplomica Verlag.

Gansel, C. \& Jürgens, F. (2007). Textlinguistik und Textgrammatik: Eine Einführung. 2., überarbeitete Auflage. Göttingen: Vadenhoeck und Ruprecht Verlag.

Gläser, R. (1990). Fachtextsorten im Englischen. Tübingen: Narr.

Grein, M. (1998). Eine typologisch-kontrastive Analyse - Mittel der Satzverknüpfung im Deutschen und im Japanischen. Wiesbaden: Springer Fachmedien Verlag.

Hentschel, E. \& Weydet, H. (2013). Handbuch der deutschen Grammatik. 4., vollständige und überarbeitete Auflage. Berlin: Walter de Gruyter Verlag.

Hilbert, S. (2014). Sprechakttheorie: Ein Überblick. Universität Hamburg: Diplomica Verlag.

Kalverkämper, H. \& Dieter Baumann, K. (Hrsg.) (1996). Fachliche Textsorten: Komponenten Relationen, Strategien. Tübingen: Narr.

Köhler, R., Altmann, G., Piotrowski, R. G. (Hrsg.) (2005). Quantative Linguistik / Quantative linguistics: Ein internationales Handbuch / An international handbook. Berlin: Walter de Gruyter Verlag. 
Mackowiak, K. (1999). Grammatik ohne Grauen: Keine Angst vor richtigen Deutsch! München: C.H. Beck.

Meibauer, J., Steinbach, M., Altmann, H. (Hrsg.) (2013). Satztypen des Deutschen. Berlin: Walter de Gruyter Verlag.

Pasch, R., Brauße, U., Breindl, E., Hermann Waßner, U. (2003). Handbuch der deutschen Konnektoren: Linguistische Grundlagen der Beschreibung und syntaktische Merkmale der deutschen Satzverknüpfer (Konjunktionen, Satzadverbien und Partikeln). Berlin: Walter de Gruyter Verlag.

Patrzek, A. (2015). Systemisches Fragen: Professionelle Fragetechnik für Führungskräfte, Berater und Coaches. Wiesbaden. Springer Gabler Verlag.

Pelz, H. (2004). Linguistik: Eine Einführung. 8., Auflage. Hamburg: Hoffmann und Campe Verlag.

Pittner, K., Bermann, J. (2007). Deutsche Syntax: Ein Arbeitsbuch. 2. Auflage. Tübingen: Günter Narr Verlag.

Dreyer, H. \& Schmitt, R. (2000). Lehr- und Übungsbuch der deutschen Grammatik. 1. Auflage. Ismaning: Max Hueber Verlag

Wachtel, M. (2005). Grammatik und vieles mehr: Linguistische Grundlagen und Lernziele für den Deutschunterricht der Sekundärstufen. 2., überarbeitete und ergänzte Auflage. Frankfurt am Main: Peter Lang Verlag.

Welke, K. (2007): Einführung in die Satzanalyse: Die Bestimmung der Satzglieder im Deutschen. Berlin: Walter de Gruyter Verlag.

\section{Internetquellen}

Das österreichische Gourmet-Portal. Chocolate cookies. Abgeufen von https://www.gutekueche.at/chocolate-cookies-rezept-7009. Abgerufen am 23.11.2019.

Das österreichische Gourmet-Portal. Karottensalat mit Chili. Abgeufen von https://www.gutekueche.at/karottensalat-mit-chili-rezept-46242. Abgerufen am 23.11.2019.

Das österreichische Gourmet-Portal. Muffin Grundrezept. Abgeufen von https://www.gutekueche.at/muffin-grundrezept-rezept-4070. Abgerufen am 10.01.2020.

Das österreichische Gourmet-Portal. Warmer Krautsalat. Abgeufen von https://www.gutekueche.at/warmer-krautsalat-rezept-4469. Abgerufen am 23.11.2019.

Deutsche Rentenversicherung. Ärztlicher Befundbericht. Abgeufen von https://bit.ly/39oF80S. Abgerufen am 28.10.2019.

Deutsche Rentenversicherung. Formulare für Ärzte im Reha-Verfahren. Abgeufen von https://bit.ly/3qiR9uO. Abgerufen am 29.10.2019.

Deutsche Rentenversicherung. Fragebogen 1 (nach Abschluss der medizinischen Rehabilitation). Abgeufen von https://bit.ly/2VbSN36. Abgerufen am 24.11.2019.

Stadtspital Waid und Triemli Zürich. APZ-Formulare (ärztl.). Abgeufen von https://bit.ly/39nmZQQ. Abgerufen am 24.11.2019.

Stadtspital Waid und Triemli Zürich. APZ-Formulare (ärztl.). Abgeufen von https://bit.ly/3mgJ4Et. Abgerufen am 24.11.2019.

WetterOnline. Wetter aktuell, Wettervorhersage und Wetterbericht. Abgeufen von https://www.wetteronline.de/wetterbericht. Abgerufen am 23.11.2019. 
WetterOnline. Wetter aktuell, Wettervorhersage und Wetterbericht. Abgeufen von https://www.wetteronline.de/wetterbericht. Abgerufen am 15.12.2019.

WetterOnline. Wetter aktuell, Wettervorhersage und Wetterbericht. Abgeufen von https://www.wetteronline.de/wetterbericht. Abgerufen am 19.12.2019. 